\title{
Feasibility of external biliary drainage in living donor liver transplantation: Lessons learned from initial experiences in single center
}

\author{
Su young HONG, Eunsung JANG, Suk Kyun HONG*, Kwangpyo HONG, Eui Soo HAN, Sanggyun SUH, \\ Jeong-Moo LEE, YoungRok CHOI, Nam-Joon YI, Kwang-Woong LEE, Kyung-Suk SUH
}

Department of Surgery, Seoul National University College of Medicine, Seoul, Korea

Introduction: Biliary complication is the most common and unmanageable complication of living donor liver transplantation (LDLT). External biliary drainage (EBD) is one of the strategies to decrease the incidence of biliary complication. Herein, we examined the LDLT cases with EBD application and evaluated the outcome of patients to assess whether EBD is a practicable surgical method to reduce biliary problem.

Methods: This study was a single-center case series study performed at Seoul National University Hospital. All adult LDLT patients with EBD application during bile duct anastomosis were included from April 2019 to May 2020. Demographic data and the rates of biliary complication and EBD-related complication were demonstrated. Median follow-up period was 15 months.

Results: EBD techniques were applied during bile duct anastomosis in 45 cases of adult LDLT patients during study period. In most cases (95.6\%), duct-to-duct anastomosis was performed. Median diameter of bile duct opening was $4 \mathrm{~mm}$ (range 1.5-16 mm). More than 2 bile duct openings were observed in 26 cases (57.8\%). With application of EBD, incidence of biliary complications was $35.6 \%$ ( $\mathrm{n}$ $=16$ ); concretely, leakage in 2 cases (4.4\%) and stricture in 14 cases (31.1\%). EBD-related complications, such as retraction, accidental removal, and dislocation, were found in 19 cases (42.2\%).

Conclusions: EBD is worthwhile in selected patients with a high risk of anastomotic bile leakage, such as multiple and/or small openings of bile duct. However, efforts should be exerted regarding EBD-related complications. Further study is needed to establish appropriate selection criteria for EBD. 